\title{
Análise do conhecimento de estudantes de uma faculdade particular do interior da
}

\section{Bahia acerca do descarte correto de medicamentos}

\author{
Analysis of the knowledge of students of a private college in the interior of Bahia about the correct \\ disposal of medicines
}

Análisis sobre el conocimiento de estudiantes de una facultad privada del interior de Bahía respecto

al correcto desecho de medicamentos

Recebido: 05/06/2021 | Revisado: 10/06/2021 | Aceito: 12/06/2021 | Publicado: 26/06/2021

\author{
Mariana de França Barros \\ ORCID: https://orcid.org/0000-0003-3563-1083 \\ Faculdade Independente do Nordeste, Brasil \\ E-mail:mafba@outlook.com \\ Carla Caroline da Silva Lima \\ ORCID: https://orcid.org/0000-0003-0547-4582 \\ Faculdade Independente do Nordeste, Brasil \\ E-mail:cacasilvalima.27@gmail.com \\ Tayanne Andrade dos Santos \\ ORCID: https://orcid.org/0000-0002-7977-6308 \\ Faculdade Independente do Nordeste, Brasil \\ E-mail: tayanneandradedossantos@gmail.com \\ Jefferson Souza Varges \\ ORCID: https://orcid.org/0000-0003-4187-4460 \\ Faculdade Independente do Nordeste, Brasil \\ E-mail: jeffersonsouzavarges@gmail.com
}

\begin{abstract}
Resumo
O descarte de medicamentos vem sendo discutido nos últimos anos, e os danos causados ao meio ambiente são a principal preocupação. Este trabalho buscou analisar o nível de conhecimento dos estudantes do curso de farmácia de uma faculdade particular do interior da Bahia, a respeito do descarte de medicamentos vencidos ou em desuso em suas residências. Trata-se de um estudo transversal, descritivo, de abordagem qualitativa e quantitativa. Para compreender como os entrevistados realizam o descarte de medicamentos vencidos ou em desuso, foi aplicado um questionário de forma online, contendo perguntas com o objetivo de identificar como são realizados os descartes dos medicamentos e saber o grau de entendimento sobre os riscos que esta prática traz. Foram entrevistados 56 alunos, $69,8 \%$ mulheres e 30,4\% homens. Destes entrevistados 91,1\% têm medicamentos armazenados em casa. Os 55,4\% dos entrevistados mantinham estes medicamentos para serem utilizados novamente e apenas 1,8\% levavam para ser descartados nas farmácias. Quando perguntados se tinham conhecimento sobre o assunto $76,8 \%$ disseram que sim, mas quando confrontados de como realizavam o descarte, 44,6\% descartavam no lixo doméstico, 23,2\% nunca descartaram, 12,5\% descartam em vaso sanitário, 5,4\% na pia, 5,3\% entregam na farmácia. Foi uma diferença grande entre o resultado de quantos tinham o conhecimento da importância do descarte correto, e os que faziam o descarte correto. Ficando claro que a é necessário estudos mais profundos relacionados ao tema sejam implantados. Para que os estudantes tenham domínio sobre o assunto, e quando profissionais possam orientar corretamente os seus pacientes em relação a importância do descarte correto dos medicamentos.
\end{abstract}

Palavras-chave: Descarte; Medicamento; Meio ambiente.

\begin{abstract}
Disposal of medications has been discussed in recent years, the damage caused to the environment is the main concern. This study aimed to analyze the level of knowledge of pharmacy students at a private college in the interior of Bahia, regarding the disposal of expired or unused medications in their homes. It was a cross-sectional study, which adopted a descriptive study, being a qualitative and quantitative research. To understand how the interviewees dispose of expired or unused medications, an online questionnaire was applied, containing questions with the aim of identifying how medications are discarded and also knowing the degree of understanding of the risks involved in this practice brings. 56 students were interviewed, $69.8 \%$ women and $30.4 \%$ men. Of these respondents, $91.1 \%$ have medicines stored at home. $55.4 \%$ of respondents kept these drugs to be used again and only $1.8 \%$ took them to be discarded in pharmacies. When asked if they were aware of the matter, $76.8 \%$ said yes, but when faced with how they were discarded, $44.6 \%$ discarded it in household garbage, $23.2 \%$ never discarded it, $12.5 \%$ discarded it in the toilet,
\end{abstract}


$5.4 \%$ at the sink, $5.3 \%$ deliver to the pharmacy. It is clear that more in-depth studies related to the topic are needed. So that students have mastery over the subject, and when professionals can correctly guide their patients regarding the importance of correct disposal of medications.

Keywords: Disposal; Medicine; Environment.

\section{Resumen}

El desecho de medicamentos viene discutiéndose en los últimos años, la principal preocupación son los perjuicios ocasionados al medio ambiente. El objetivo de este estudio fue analizar el nivel de conocimiento de los estudiantes de farmacia, de una facultad privada del interior de Bahía, sobre cómo deshacerse en sus hogares de medicamentos vencidos o sin uso. Se trata de un estudio de modelo transversal, que adoptó un estudio descriptivo, siendo una investigación cualitativa y cuantitativa. Para entender cómo los encuestados desechan los medicamentos vencidos o sin uso, se aplicó un cuestionario online, cuyas preguntas objetivaban identificar cómo desechan los medicamentos y también conocer su grado de comprensión sobre los riesgos que conlleva esa práctica. Se entrevistaron 56 estudiantes, $69,8 \%$ mujeres y 30,4\% hombres. De estos encuestados, el 91,1\% tiene medicamentos almacenados en casa. El 55,4\% de los encuestados mantenían estos medicamentos para volver a usarlos y solo el 1,8\% los llevaban para desechar en las farmacias. Cuando se les preguntó si tenían conocimiento sobre el tema, el 76,8\% respondió que sí, pero sobre la manera de desecharlos, el 44,6\% los desechaba en la basura de su casa, el 23,2\% nunca los desechaban, el 12,5\% los desechaban en el inodoro, el 5,4\% en el fregadero, el 5,3\% los entregaban en la farmacia. Queda claro que es necesario implantar estudios más profundos sobre este asunto. Para que los estudiantes dominen el tema y cuando actúen como profesionales puedan orientar a sus pacientes sobre la importancia de desechar correctamente los medicamentos.

Palabras clave: Desechar, Medicamento, Medio Ambiente.

\section{Introdução}

O crescimento da população trouxe como consequência o aumento dos tipos de enfermidades e consequentemente o grande consumo e armazenamento de medicamentos. Dessa forma a maioria da população não recebe as instruções adequadas para realizar o descarte correto dessas substâncias que estão presentes em suas residências e assim descartam os seus medicamentos em locais inapropriados como lixo comum, dentro de pias e vasos sanitários (Gonzales \& Ferreira, 2020).

O excesso de medicamentos em suas residências também é um fator que favorece o descarte inadequado de medicamentos. $\mathrm{O}$ acúmulo de medicamentos em suas residências é um ato comum na cultura brasileira, visto que a população recebe incentivos da mídia para a aquisição desses medicamentos e assim gerando o acúmulo dessas substâncias em suas residências (Fernandes \& Petrovi, 2004).

Dessa forma a atuação do profissional farmacêutico no ato da dispensação de medicamentos é de suma importância, pois ele irá ajudar a diminuir o número de medicamentos dispensados, realizando uma dispensação adequada para o paciente com o intuito de não ocorrer o acúmulo e o uso indiscriminado desses medicamentos (Guirguis, 2010).

Atualmente não existe uma lei para regulamentar o descarte final de medicamentos para a população em geral, somente para os estabelecimentos de saúde, o que dificulta a realização do controle total de medicamentos descartados e ocasionando a dificuldade da preservação do meio ambiente e da saúde pública (Ueda et al., 2009).

O descarte inapropriado de medicamentos apresenta vários riscos para o meio ambiente, porém o risco que mais se destaca é a contaminação dos recursos hídricos que provocam o surgimento de diversas doenças na população e a extinção de diversas espécies de animais e vegetais. Outra questão é a resistência bacteriana, pois esse resíduo poderá entrar em contato com diversas bactérias e assim ocasionando alteração no seu material genético e tornando a bactéria resistência ao medicamento (Balbino \& Balbino, 2017).

Frente ao exposto, este trabalho teve por objetivo identificar como os alunos de uma faculdade do interior da Bahia realizam o descarte de medicamentos e o seu conhecimento sobre os malefícios que este descarte incorreto traz para a população. 


\section{Metodologia}

O presente estudo terá um modelo transversal com estudo descritivo, sendo uma pesquisa quantitativa e qualitativa. Foi realizada no município de Vitória da Conquista (BA). Para a realização da coleta dos dados utilizou-se um questionário online, tendo a instituição de ensino disponibilizado os e-mails dos estudantes do curso de farmácia e assim o questionário foi encaminhado para os e-mails. Foi criado um e-mail exclusivo para o encaminhamento do questionário para os entrevistados, os dados foram utilizados apenas para o propósito da pesquisa. Estava presente no questionário perguntas com respostas fechadas com o intuito de otimizar e facilitar as respostas do entrevistado, e com perguntas com respostas abertas, pois possibilita respostas ricas, variadas e mais descritivas (Basto \& Duquia, 2007).

A coleta foi realizada no período de março a maio de 2021, com os alunos devidamente matriculados do curso de farmácia da instituição. Aqueles entrevistados que concordaram, receberam um material socioeducativo no seu e-mail para compreender como realizar o descarte correto dos medicamentos vencidos ou em desuso e se conscientizar para realizar o descarte adequado.

Os dados adquiridos a partir desta pesquisa foram dispostos e compilados em um banco de dados utilizando o programa Microsoft Office Excel® 2010. Sua interpretação foi baseada na análise estatística descritiva contendo os resultados do questionário e apresentado por meio de gráficos e porcentagem dos resultados da pesquisa.

O desenvolvimento da pesquisa atendeu aos princípios éticos que constam na resolução $\mathrm{N}^{\circ} 466$, de 12 de dezembro de 2012 do Conselho Nacional da Saúde, sendo aprovado pelo Comitê de Ética em Pesquisa da Faculdade Independente do Nordeste (CEP/FAINOR) sob parecer $\mathrm{N}^{\circ}$ 4.585.480, foi feito o Termo de Consentimento Livre e Esclarecido entregue a todos os participantes da pesquisa, o entrevistado teve a sua identidade com padrões profissionais de sigilo, utilizando as informações somente para fins acadêmicos e científicos prevalecendo o bem-estar e a saúde da população de acordo com a declaração de Helsinque.

\section{Resultados e Discussão}

Participaram da pesquisa 56 pessoas com idade entre 18 e 40 anos. Cerca de 58,9\% dos pesquisados tem de 21 a 25 anos de idade, se tornando o número predominante. Outros 21,4\% têm entre 18 e 20 anos, $10,7 \%$ entre 26 e 30 anos e em menor quantidade os maiores de 30 anos com 8,9\%. Segundo o Ministério da Educação, as matrículas de jovens com idade de 19 a 24 anos no ensino superior são cerca de 56,45\%. Reforçando o pensamento de que os jovens têm cada vez mais se interessado ao nível superior (MEC, 2017).

Na pesquisa realizada, o sexo predominante dos participantes foi de mulheres, tendo o percentual de $69,8 \%$, e os homens com a porcentagem de 30,4\%. Indo a favor dos dados ofertados pelo INEP de que 57,2\% dos alunos do ensino superior são mulheres, segundo o último censo de 2016. O Conselho Federal de Farmácia em 2015 desta que as profissionais farmacêuticas eram de $67,5 \%$, sendo assim a maioria. O que dá uma ênfase no número de estudantes do sexo feminino no curso de farmácia (CFF, 2015; INEP, 2018).

Dos alunos entrevistados $71,4 \%$ dizem fazer uso de medicamentos continuamente. $\mathrm{O}$ anticoncepcional foi equivalente a $20 \%$ das respostas, indo concordância com o número maior de mulheres da pesquisa. Cerca de $79 \%$ das brasileiras fazem o uso de anticoncepcional, como forma de prevenção de uma gravidez, o segundo mais citado foram os suplementos vitamínicos com $12,5 \%$. Devido à alimentação pobre em nutriente que tem aumentado na última década e a preocupação em ter uma vida saudável e prolongada, a procura pelos suplementos tem aumentado para fazer esta reposição. Outros medicamentos citados foram reposição hormonal para problemas de tireoide, anti-hipertensivo, diuréticos, anticonvulsivante, corticoide, anticoagulante, inibidores de bomba de prótons e anti-inflamatório (Matsumoto, 2015; Ribeiro et al., 2018). 
O fácil acesso aos medicamentos e a prática cada vez mais comum da automedicação vem fazendo com que o número das famosas "farmacinhas domiciliar" cresça. Em sua grande maioria, 91,1\% dos participantes dizem ter medicamentos armazenados em casa, e apenas 8,9\% dizem não ter. Resultado similar foi encontrado na pesquisa de Bueno et al. (2009) dizendo que 91,59\% dos entrevistados diz ter a farmácia caseira em suas residências. No estudo de Ferreira et al. (2015) em Divinópolis, MG, a presença de medicamentos foi observada em 96,6\% dos domicílios da região de seis UBSs, ou seja, esses resultados confirmaram que grande parte da população possui estoques de medicamento em suas residências o que facilita que o paciente realize a automedicação (Fernandes et al., 2019).

Um dos motivos para ocorrer o estoque de medicamentos em suas residências são as sobras de prescrições anteriores, a falta de adesão ou a troca do tratamento estão entre elas. No estudo de Vatovec et al. (2017) foi relatado que o motivo mais comum pelos quais os entrevistados terem sobras de medicamentos foi que eles usaram o medicamento até se sentirem melhor e então pararam de usá-lo (Constantino et al., 2018).

Conforme dados da Tabela 1, os entrevistados foram questionados quanto à forma de armazenamento dos medicamentos em casa e disseram que guardavam no quarto $62,5 \%$, na cozinha $35,7 \%$, na sala $10,7 \%$ e nenhum dos participantes relataram guardar no banheiro.

Tabela 1. Local que costuma armazenar os medicamentos.

\begin{tabular}{cc}
\hline LOCAL & PORCENTAGEM \\
\hline Quarto & $62,5 \%$ \\
Cozinha & $35,7 \%$ \\
Sala & $10,7 \%$ \\
Banheiro & - \\
\hline
\end{tabular}

Fonte: Autores (2021).

O mesmo ocorreu na pesquisa de Gonzales e Ferreira (2020) que entre os locais de armazenamento, os quartos $(41,8 \%)$ e as cozinhas $(36,1 \%)$ foram os mais citados entre os participantes. Muitas pessoas escolhem esses locais por serem de fácil acesso e fazer parte do seu cotidiano. Este é um problema preocupante, pois a cozinha é o local mais citado na pesquisa, onde o calor e a temperatura variam, não havendo proteção contra calor, umidade e ventilação (Dutra et al., 2018).

O local de armazenamento influencia na qualidade dos medicamentos por interferir nas características físicoquímicas, por isso devem ser guardados em lugares arejados, sem exposição à luz e a umidade, nas suas embalagens originais mantendo os dados como data de vencimentos e princípio ativo. Além disso, é de suma importância o cuidado em manter estas substâncias fora do alcance de crianças evitando acidentes de intoxicação por ingestão delas pelos menores (Cruz et al., 2017).

$\mathrm{Na}$ Tabela 2 traz dados dos entrevistados quanto à finalidade dos medicamentos em desuso. $\mathrm{O}$ ato de armazenar os medicamentos é uma prática sem segurança garantida, as pessoas não costumam descartar os medicamentos que está em desuso e com consequência realiza o armazenamento destes insumos com a finalidade de utilizar em outra ocasião. O número de respostas da pesquisa que guardavam o medicamento para usar outra vez foi de $55,4 \%$, realiza o descarte no lixo $23,2 \%$, não sobra medicamento 12,5\% doam para vizinhos, amigos ou parentes 1,8\% e leva para farmácia 1,8\% (Athern et al., 2016). 
Tabela 2. O que é feito com os medicamentos em desuso.

\begin{tabular}{cc}
\hline FINALIDADE & PORCENTAGEM \\
\hline Guarda para usar outra vez & $55,4 \%$ \\
Realiza o descarte no lixo & $23,2 \%$ \\
Não sobra o medicamento (Utiliza todo) & $12,5 \%$ \\
Doa para vizinhos, amigos, parentes. & $1,8 \%$ \\
Leva para a farmácia & $1,8 \%$ \\
\hline
\end{tabular}

Fonte: Autores (2021).

Estes resultados se assemelham a pesquisa realizada por Gonzales e Ferreira (2020), onde $48 \%$ dos entrevistados afirmaram que realiza o descarte dos medicamentos, enquanto $47,1 \%$ afirmaram que guardam as sobras de medicamentos em suas residências. Já no estudo de Bueno et al. (2009), 36,6\% dos entrevistados guardam as sobras dos medicamentos para utilizar em uma outra ocasião no futuro. Se diferenciando um pouco dos resultados desta pesquisa

Foi observado que 55,4\% dos participantes da pesquisa dizem consumir os medicamentos que sobram e 44,5\% dizem não fazer esta prática. Dentre esses medicamentos $57,1 \%$ dos medicamentos em casa são para o caso de emergência, 55,4 \% por facilitar o uso, 19,6\% fazem o uso de maneira contínua do medicamento, 16,1\% diz ser sobras de tratamentos prescritos, $8,9 \%$ evitar a ida ao médico, $3,6 \%$ por se esquecer de descartar e 1,8\% relata ter recebido de um conhecido. No estudo de Zorpas Dimitriou e Voukkali (2018) os dados foram semelhantes, pois cerca de 8,2\% dos entrevistados mencionaram que realizava a doação de medicamentos não utilizados para seus amigos ou para um familiar por considerar que esses medicamentos sem uso são úteis. Os medicamentos compartilhados também fazem parte da automedicação, por ser uma forma de tratamento sem acompanhamento de um profissional pode trazer risco a saúde. Esta prática pode causar resistência bacteriana, intoxicação medicamentosa e o mascaramento da doença (Morais \& Júnior, 2018).

$\mathrm{O}$ ato de armazenar medicamentos que está em desuso nas residências é um fator de risco para a saúde da população, pois ocorre uma possibilidade de intoxicação provocada pelo consumo desses insumos através da automedicação e pode ocorrer a diminuição da eficiência e segurança dos medicamentos causada pela falta de cuidados com a farmácia caseira (Ferreira et al., 2015).

Apesar da preocupação com a finalidade dos resíduos medicamentosos, os estudos e programas que asseguram o descarte seguro destes insumos ainda são poucos. A orientação que se tem é que deve ser feito a devolução do medicamento em desuso nas drogarias e estabelecimentos de saúde que tenham o PGRSS (Ramos et al.,2017). Porém nesse estudo nem todos tem essa informação, 76,8\% dos entrevistados foram orientados em relação ao descarte do medicamento e 23,3\% dos entrevistados relatou não ter nenhum tipo de orientação de como realizar o descarte de medicamentos. Dos entrevistados $76,74 \%$ disseram ter tido a orientação de como realizar o descarte de medicamentos em sua faculdade durante o curso e os outros $23,26 \%$ relataram que recebeu a orientação de como descartar os medicamentos nas farmácias ou em cursos que fizeram.

A maioria dos entrevistados, $87,5 \%$, disseram se preocupar com o descarte correto dos medicamentos. Porém, as respostas relacionadas ao descarte preocupam, pelo fato de não serem feitos de maneira correta. No estudo de Gonzales e Ferreira (2020), todos os entrevistados consideraram que é importante realizar o descarte de medicamentos em local específico, embora não conheçam as instituições que aceitam esses produtos para facilitar o descarte adequado, o que indica falta de infraestrutura e de acesso da população.

A Tabela 3 traz a informação de como os entrevistados fazem os descartes dos seus medicamentos. As respostas mais 
frequentes sobre o descarte de medicamentos foram lixo doméstico 44,6\%, nunca descartou 23,2\% e o vaso sanitário $12,5 \%$, tendo outras respostas com valores abaixo de dez por cento. Não condizendo com a informação de que a maioria deu dizendo se preocupar com o descarte correto. Se tornando um conflito entre teoria e prática que é ruim e causa danos a saúde dos seres vivos e do meio ambiente.

Tabela 3. Como é realizado o descarte dos medicamentos.

\begin{tabular}{cc}
\hline LOCAL DE DESCARTE & PORCENTAGEM \\
\hline Lixo doméstico & $44,6 \%$ \\
Nunca descartou & $23,2 \%$ \\
Vaso sanitário & $12,5 \%$ \\
Pia & $5,4 \%$ \\
Entrega na farmácia & $5,3 \%$ \\
Coloca fogo & $3,6 \%$ \\
Deixa em casa & $1,8 \%$ \\
Unidade de Saúde & $1,8 \%$ \\
Posto de descarte & $1,8 \%$ \\
\hline
\end{tabular}

Fonte: Autores (2021).

No estudo realizado por Constantino et al, (2018), as formas de descarte de medicamentos em domicílio são, no lixo comum, rede de esgoto, diretamente ao solo, colocando fogo e algumas pessoas devolviam para os estabelecimentos. Se assemelhando aos resultados obtidos pela pesquisa, na qual a maioria da forma de descarte também é no lixo e vaso sanitário. A maneira como os descartes acontecem é um desafio mundial, porque quando descartados no lixo comum, ou no vaso sanitário podem trazer malefícios a saúde humana. Por serem compostos biológicos, a degradação do fármaco é rápida e fácil, e quando entram em contato com o meio causa danos as várias formas de vida do ambiente. Desta maneira os descartes destes insumos devem ser de maneira consciente e no lugar correto, para que os danos ao meio ambiente sejam amenizados (Constantino et al., 2018; Sousa et al., 2020).

\section{Conclusão}

Os resultados da pesquisa mostram que mesmo sendo estudantes do curso de farmácia, os conhecimentos relacionados ao descarte de medicamentos ainda são escassos. Apesar de 87,5\% dos participantes dizerem se preocupar com este tema, ainda realizam os descartes de maneira incorreta.

Os dados coletados demonstram uma fragilidade no conhecimento e prática do público entrevistado sobre os impactos causados ao meio ambiente, quando estes produtos são expostos de forma inadequada.

Desta forma, é necessário que estudos mais profundos relacionados ao tema sejam implantados, para que os estudantes tenham domínio sobre o assunto, e quando profissionais possam orientar corretamente os seus pacientes em relação a importância do descarte correto dos medicamentos.

Novos estudos poderão ser desenvolvidos para análise da melhor maneira de ensinar sobre a importância do descarte correto dos medicamentos e seus impactos ao meio ambiente. E a partir de um projeto de educação sanitária em relação ao descarte dos medicamentos, fazer um estudo dos resultados para saber se foram eficazes os ensinamentos. 


\section{Referências}

Athern, K. M., Linnebur, S. A., \& Fabisiak, G. (2016). Proper Disposal of Unused Household Medications: The Role of the Pharmacist. The Consultant pharmacist: the journal of the American Society of Consultant Pharmacists, 31(5), 261-266. 10.4140/TCP.n.2016.261

Balbino, M. L. C., \& Balbino, E. C. (2017). O descarte de medicamentos no Brasil: Um olhar socioeconômico e ambiental do lixo farmacêutico. Revista Brasileira De Estudos Jurídicos-Faculdades Santo Agostinho, 7, 1, 87-100.

Bastos, D. L.J., \& Duquia, P. R. (2007). Um dos delineamentos mais empregados em epidemiologia: estudo transversal. Scientia Medica, Porto Alegre, 17, 4, 229- 232 .

Cruz et al. (2017). Estoque doméstico e uso de medicamentos por crianças no Vale do Jequitinhonha, Minas Gerais, Brasil. Saúde Debate, 41 (114), 836-847. doi: 10.1590/0103-1104201711413

Constantino et al. (2018). Estoque e descarte de medicamentos no domicílio: uma revisão sistemática. Ciência \& Saúde Coletiva, 25(2):585-594.

Chien, Y. H., Ko, J. J., Chen, Y. C., Weng, H. S., Yang, C. W., Chang, C. Y., \& Liu, P. H. (2013). Estudo de resíduos de medicamentos em Taiwan. J Exp Clin Med 5 (2): $69-72$.

Diretoria de Estatísticas Educacionais - Deed. (2018). Ministério da Saúde: MEC Censo de educação superior do ano de 2017. http://portal.mec.gov.br/index.php?option=com_docman\&view=download\&alias=97041-apresentac-a-o-censo-superior-u-ltimo\&Itemid=30192.

Dutra, K. R. et al. (2018). Condições de armazenamento de medicamentos nas Unidades Básicas de Saúde do Distrito Sanitário Nordeste de Belo Horizonte (MG), Rev Infarma, 30, 2, 130-13.

Ferreira, C. L., \& Rodrigues, S. C. (2015). Análise do conhecimento da população sobre descarte de medicamentos em Belo Horizonte/mg. Interfaces Científicas, Saúde e Ambiente, 3(2): 9-18.

Fernandes, M. R., Figueiredo, R. C. D., Silva, L. G. R. D., Rocha, R. S., \& Baldoni, A. O. (2020). Armazenamento e descarte dos medicamentos vencidos em farmácias caseiras: problemas emergentes para a saúde pública. Einstein, 18.

Fernandes, L. C., \& Petrovick. P. R. (2004). Os medicamentos na farmácia caseira. In: Schenkel EP. Cuidados com os medicamentos. Editora da UFRGS; 3942 .

Guirguis, K. (2010). Medications collected for disposal by outreach pharmacists in Australia. Pharm World Sci, 32(1), 52-58.

Gonzales, G.; Ferreira, E. (2020). Percepção de Universitários de Campo Grande (MS) Sobre o Descarte de Medicamentos Domiciliares e seus Impactos ao Meio Ambiente. Rev. Cienc. Gerenc, 24, 4, 370-380.

Matsumoto, L., Sampaio, G., \& Bastos, D. (2015). Suplementos vitamínicos e/ou minerais: regulamentação, consumo e implicações à saúde. Cad. Saúde Pública, 31(7), 1371-1380.

Mapa do Ensino Superior aponta maioria feminina e branca. (2020, Maio 21). Mapa do Ensino Superior aponta maioria feminina e branca. Agência Brasil. https://agenciabrasil.ebc.com.br/educacao/noticia/2020-05/mapa-do-ensino-superior-aponta-para-maioria-feminina-e-

branca\#: :text=Mais\%20da\%20metade\%20das\%20matr\%C3\%ADculas,est $\%$ C3\%A1\%20na\%20faixa\%20dos\%2018\%25

Morais, E., \& Furlan, O. (2018). Consequências E Quais Os Principais Riscos Da Automedicação. https://www.unifacvest.edu.br/assets/uploads/files/arquivos/e692c-ereni-de-morais---consequencias-e-quais-os-principais-riscos-da-automedicacao.pdf.

Ramos, H. M. P., Cruvinel, V. R. N., Meiners, M. M. M. D. A., Queiroz, C. A., \& Galato, D. (2017). Descarte de medicamentos: uma reflexão sobre os possíveis riscos sanitários e ambientais. Ambiente \& Sociedade, 20(4).

Ribeiro, C. C. M., Shimo, A. K. K., Lopes, M. H. B. M., \& Lamas, J. L. T. (2018). Effects of different hormonal contraceptives in women's blood pressure values. Rev Bras Enferm, 71, 1453-9.

Sousa, P. V. A., Sousa, M. S., Sousa, G. S., Souza, O. G. B., \& Santos, T. S. (2020). Effects of drug disposal on the environment. Research, Society and Development, 9(7): 1-11.

Ueda, et al. (2009). Impacto Ambiental do descarte de Fármacos e estudo da conscientização da população a respeito do problema. Revista ciência do ambiente. 5, 1 .

Vatovec, C., Van Wagoner, E., \& Evans, C. (2017). Investigating sources of pharmaceutical pollution: Survey of over-the counter and prescription medication purchasing, use and disposal practices among university students. Journal of environmental management, 198, 348-352.

Zorpas, A. A., Dimitriou, M., \& Voukkali, I. (2018). Disposalo fhouse hold pharmaceuticals in insular communities: social attitude, behaveou revaluation and prevention activities. Environmental Science and Pollution Research, 25(27): 26725-26735. 